\title{
Linear immunoglobulin a dermatosis-a less reported clinicopathologic entity
}

\begin{abstract}
Linear immunoglobulin A dermatosis (LAD) is a rare autoimmune vesiculobullous disease with an incidence of 0.5 per million in west Europe but more common in China, Southeast Asia and Africa. It is commonly confused with dermatitis herpetiformis and differentiated based on the pattern of Immunoglobulin A (IgA) deposition on skin biopsy. In India it is rarely reported and literature states the first report was in $1997 .{ }^{1}$ LAD can affect people of all races and theoretically, all ages, but two peaks have been observed: childhood-onset and adult-onset. Herein we describe an adult type of LAD who recovered from LAD but later succumbed to secondary infections.
\end{abstract}

Keywords: autoimmune, bullous eruptions, immunoglobulin a, linear iga
Volume 5 Issue 4 - 2017

\author{
Chenna KeshavaBG,' Sridhar, ${ }^{2}$ Padmini P \\ Giri, ${ }^{3}$ Padmini P Giri' \\ Department of Critical Care, MS Ramaiah hospitals, Bangalore, \\ India \\ 2Department of Pathology, MS Ramaiah hospitals, Bangalore, \\ India \\ ${ }^{3}$ Department of Medicine, MS Ramaiah hospitals, Bangalore, \\ India
}

Correspondence: Chenna Keshava BG, Consultant Intensivist, Department of Critical Care, MS Ramaiah hospitals, Bangalore, India,Tel 988-605-159-8,Email ckeshava7@gmail.com

Received: January 01, 1970 | Published: May 17, 2017
Abbreviations: DH, dermatitis Herpetiformis; BA, bronchial asthma; DIF, direct immunofluorescence; HTN, hypertension; Iga, immunoglobulin A; LAD, linear immunoglobulin a dermatosis; NIV, non-invasive ventilation

\section{Introduction}

Linear IgA disease (LAD) is a chronic, acquired, autoimmune bullous disease. Generalized bullous eruptions is not unusual in the intensive care unit. The cause can be idiopathic, autoimmune, or drug induced but prompt recognition is crucial to their management. The autoimmune blistering skin diseases are a heterogeneous group of diseases associated with autoantibodies that are directed against desmosomal structural proteins (in pemphigus diseases) or hemidesmosomal ones (in pemphigoid diseases and epidermolysis bullosa acquisita), or else against epidermal/tissue transglutaminases (dermatitis herpetiformis or LAD). Dermatitis herpetiformis (DH) and LAD are IgA mediated autoimmune bullous diseases and shares the same histopathology characters. They are differentiated based on the pattern of $\operatorname{Ig} \mathrm{A}$ deposition on direct immunofluorescence. Herein we describe an adult patient with LAD who recovered from LAD but later succumbed to secondary infections.

\section{Case presentation}

This was a 74-year old male patient known case of hypertension (HTN) and Bronchial Asthma (BA) on regular treatment, presented with complaints of facial swelling, breathlessness and skin lesions since five days. He developed breathlessness-grade 2, but gradually progressed to breathlessness at rest by the time of admission which was not relieved with inhaler. His skin lesions (vesicles) initially appeared on lips and later on hands and legs. His history was positive for swelling of lower limbs followed by puffiness of the face. His immediate past history was significant for fever and joint pains, which started three months ago, suspected to be viral arthritis and was prescribed steroids and painkillers by a local doctor, which he has been taking on and off. He denied history of fever, nausea, vomiting, loose motions, or decreased urine output.

On examination his pulse was $90 \mathrm{bpm}$, blood pressure was 110/70 mmhg, respiratory rate was $4 \mathrm{cpm}$, saturations of $97 \%$ on 8 litres oxygen and afebrile. His systemic exam showed bilateral pitting pedal edema, periorbital edema present and occasional rhonchi heard bilaterally on lung auscultation. All other systems were essentially normal. His had multiple vesicles and bullae over the extensor aspect of both upper limbs (including palms), groin, buttocks and lower abdomen (Figure 1A \& B). The patient was admitted to intensive care unit (ICU) with a provisional diagnosis of acute exacerbation of bronchial asthma with hypertension, probably herpes labialis versus kaposi's varicelliform eruptions (KVE). He was started on injection acyclovir and ceftriaxone with supportive management. His initial investigations were as in Table 1. All reports were within normal limits except for a mild elevation in serum creatinine. Chest $\mathrm{x}$-ray was normal. The patient's 2D ECHO showed mild PAH with grade 1 left ventricular diastolic dysfunction. USG abdomen showed mild right pleural effusion with kidneys showing normal cortical echotexture. Urine routine showed 15-20 WBC and no RBC's.

Over the first 24 hours he had a worsening of tachypnea with acidosis (pH of 7.09) and hypotension. He was started on intermittent non-invasive ventilation (NIV), and inotropes. Nephrology opined and in view of the low urine output, worsening acute kidney injury; he underwent hemodialysis. Due to increasing total white cell count, antibiotics were escalated to meropenem. Dermatology evaluated and opined for the skin lesions and collected specimens for tzanck smear, histopathology examination (HPE) (Figure 2 A-C) and direct immunofluorescence (DIF). DIF revealed linear IgA deposits confirming the diagnosis of IgA Dermatosis. Patient was started on injection methylprednisolone 500gm once a day. Due to worsening saturations and tachypnea patient was electively intubated on the evening of day 2. The patient was weaned and extubated on D8 of ICU stay. His creatinine improved with adequate urine output after the initial dialysis. On day 14 he was started on injection teicoplanin and colistin nebulisation, empirically due to a sudden surge in white cell count. The patient's wound and sputum cultures taken on D13 was positive for acinetobacter baumannii and Klebsiella pneumoniae respectively and was started on appropriate antibiotics based on culture sensitivity. The patient was shifted from the ICU to the wards on Day 16. On Day 21 patient was shifted back to ICU due to severe breathlessness. He was intubated and ventilated on emergency basis. On Day 25 of hospitalization, the patient was declared dead due to severe gram negative sepsis. 


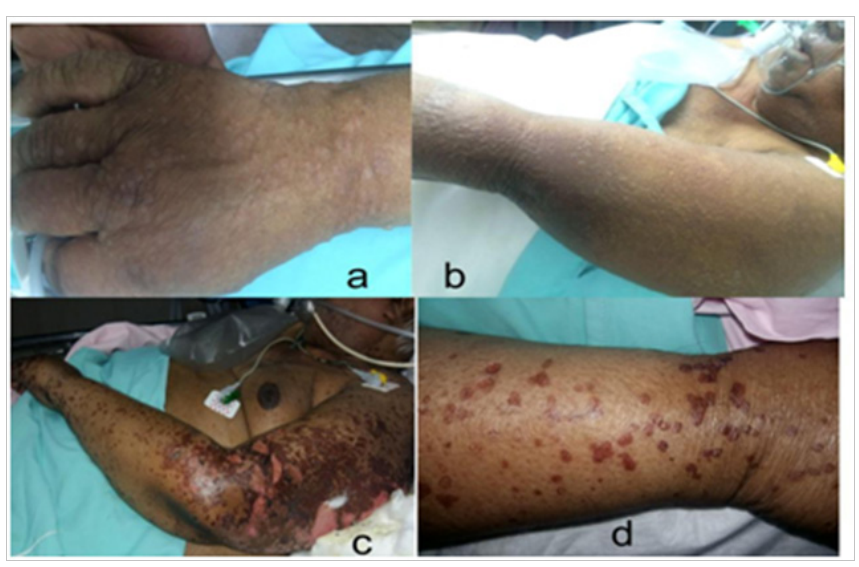

Figure I A \& B shows Tense vesicles at admission on dorsum of hand, arm and upper forearm. C \& D shows Resolving phase of blisters.

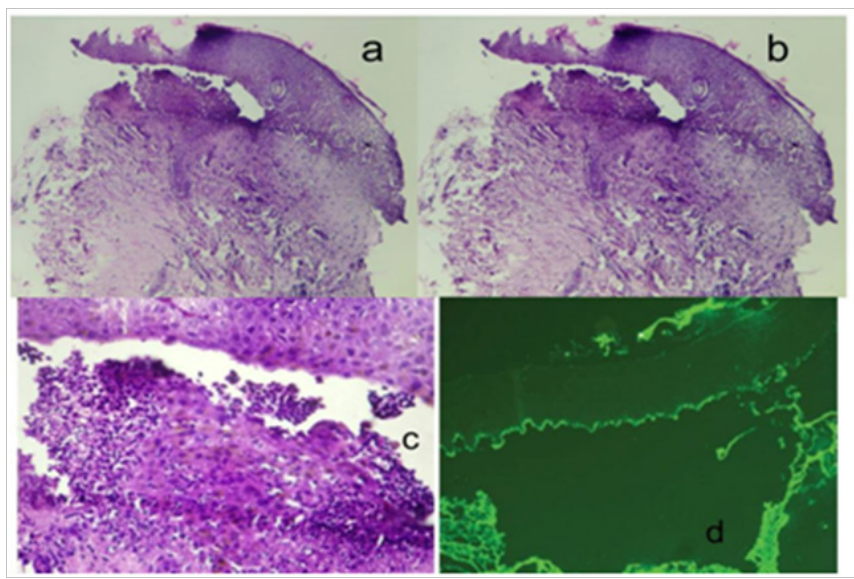

Figure 2A.B,Cshows subepithelialsplitand inflammatoryinfiltrateinconnective tissueskintissuewithsubepidermalblisterand neutrophilsandfibrinin theirlumen. $\mathrm{d}$ shows DIF image showing linear deposition of immunoglobulin $\mathrm{A}(\lg \mathrm{A})$ at the basement membrane (The original DIF image could not be displayed due to logistical reasons. ${ }^{8}$
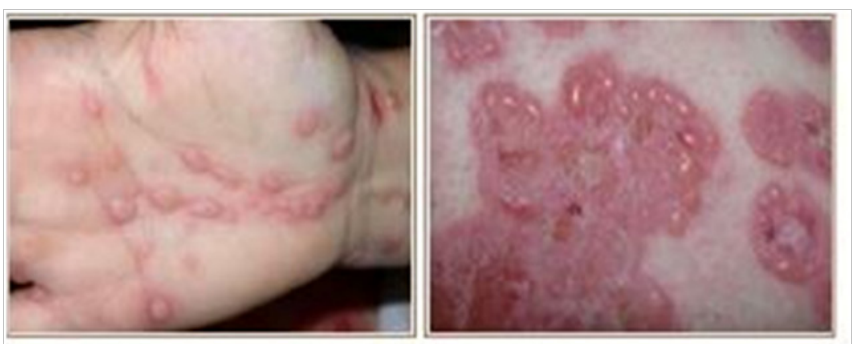

Figure 3 String of beads.

\section{Discussion}

Linear immunoglobulin A dermatosis or Linear immunoglobulin A bullous dermatosis, also known in literature as linear IgA dermatosis, linear IgAdisease, IgA pemphigoid, and linear dermatitis herpetiformis, was first described by Bowen in 1901. It was recognized as a distinct entity from dermatitis herpetiformis in 1979. The childhood variant usually appears after age 6 months (several newborns with LAD have been described) and before the age of 5 or 6 (mean 3.3-4.5) years, rarely persisting after puberty. The adult-onset appears after puberty or later in life, generally after age 60 . Epidemiologic data on its distribution and sex ratio worldwide are very heterogeneous for both variants. ${ }^{2}$ In adults the disease is more protracted with less remissions than children $(48 \%))^{3}$ It is a non-scarring disease and tends to wax and wane in severity. Though many cases are idiopathic, the commonly implicated causes are drugs, internal malignancies, sepsis, skin trauma, gastrointestinal disease etc. Vancomycin is the drug most commonly implicated, diclofenac and other nonsteroidal anti inflammatory drugs being less commonly reported. ${ }^{4}$ All studies on genetic linkage to LAD have not been fruitful and it is found that no ethnic predilection or susceptibility has ever been reported. There is a strong association between LAD and the extended autoimmune haplotype HLA-B8, HLA-CW7, HLA-DR3 in British and black South African patients, and possession of this haplotype is associated with an early disease onset. This extended haplotype is in linkage disequilibrium with an activating allele of tumor necrosis factor (TNF), and this association exists in patients with LAD and seems to worsen prognosis and prolong the disease. ${ }^{5}$

The clinical features of LAD in children are different when compared to adults. They usually present with vesicles, blisters or/ and erosions involving skin and mucous membranes. Lesions can be vesicles (or both) or bullae, with clear or hemorrhagic fluid, that have risen out of normal skin, sometimes with an erythematous or urticarial base. ${ }^{6}$ Bullae or vesicles are generally tense, vary in size, and frequently tend to form annular or polycyclic plaques due to the coalescence of lesions, with a characteristic blistering along the edge of the lesion, the so-called "string-of- pearls" sign (Figure 3). The distribution of lesions in children or adults can be symmetric or asymmetric. They have a polymorphic pattern with unusual features, such as itchy, roundish excoriated papules or nodules (or both), typical of prurigo nodularis or contact dermatitis like lesions or as urticarial papules. Any mucous membranes can be involved but most commonly affects oral cavity (hard and soft palate, palatine arches, and buccal mucosa) and eyes. Ocular LAD may be uni/bilateral chronic conjunctivitis that may result in a cicatricial conjunctivitis, with or without subconjunctival fibrosis and symblepharon formation.

The reason for this subepidermal blisters, caused by IgA antibodies against target proteins in the epidermal adhesion complex. The major target antigen is BP180/collagen XVII, a key structural component of the dermoepidermal adhesion complex. The intracellular BP230, initially found to be a major antigen in bullous pemphigoid, is also targeted by IgA antibodies, more commonly in adult LAD. The pathophysiologic mechanism of drug-induced LAD still remains largely unknown. The diagnosis of LAD is mainly based on clinical, histopathology and immunologic. ${ }^{7}$. It is difficult to distinguish LAD from other autoimmune cutaneous blistering disease. By clinical and histologic features alone, due their frequent overlap. The presence of IgA autoantibodies using immunopathologic examinations like DIF, indirect immunofluorescence, Western immunoblotting or immunoelectron microscopy. The mainstay of treatment is dapsone, steroids and general supportive care for the bullae. Dapsone and sulfapyridine as monotherapy are the preferred first and second line agents except in severe cases of drug induced LAD wherein a short course of steroids is considered. Our patient was an elderly male, treated frequently with steroids and pain killers for arthritis. The pain killers included a wide array of NSAID's including diclofenac, which could have been the precipitating factor. An early biopsy and DIF helped us in a quick diagnosis and early initiation of steroids. Though he had a good initial and was shifted out of hospital he succumbed to hospital acquired infections secondary to multidrug resistant organism. 


\section{Conclusion}

LAD though a rare entity should be a part of differential diagnosis of any pruritic, urticarial dermatitis with or without a bullous lesions and is resistant to topical treatments. Though LAD is a rare entity, a high index of suspicion and early biopsy with DIF helps in accurate diagnosis and appropriate treatment. An early steroid initiation in severe cases is recommended to control the disease process and the immune damage.

\section{Acknowledgments}

None.

\section{Conflicts of interest}

The authors declare no conflicts of interest.

\section{References}

1. Ajithkumar K, Kurian S, Jacob M, et al. Linear IgA bullous dermatosis in South India. International Journal of Dermatology. 1997;36(3):191193.

2. Fortuna G, Marinkovich M. Linear immunoglobulin A bullous dermatosis. Clinics in Dermatology. 2012;30(1):38-50.
3. Chanal J, Ingen-Housz-Oro S, Ortonne N, et al. Linear IgA bullous dermatosis:comparison between the drug-induced and spontaneous forms. British Journal of Dermatology. 2013;169(5):1041-1048.

4. McDonald H, York N, Pandya A. Drug-induced linear IgA bullous dermatosis demonstrating the isomorphic phenomenon. Journal of the American Academy of Dermatology. 2010;62(5):897-898.

5. Collier P, Wojnarowska F, Welsh K, et al. Adult linear IgA disease and chronic bullous disease of childhood:the association with human lymphocyte antigens $\mathrm{Cw} 7, \mathrm{~B} 8, \mathrm{DR} 3$ and tumour necrosis factor influences disease expression. British Journal of Dermatology. 1999;141(5):867-875.

6. Antiga E, Caproni M, Fabbri P. Linear Immunoglobulin A Bullous Dermatosis:Need for an Agreement on Diagnostic Criteria. Dermatology. 2013;226(4):329-332.

7. Venning V. Linear IgA Disease:Clinical Presentation, Diagnosis, and Pathogenesis. Immunology and Allergy Clinics of North America. 2012;32(2):245-253.

8. Joseph T, Sathyan P, Goma Kumar K. Linear IgA dermatosis adult variant with oral manifestation:A rare case report. Journal of Oral and Maxillofacial Pathology. 2015;19(1):83-87. 